\title{
Covid-19, the Climate Change Crisis and Breakdown of the Global Markov Blanket
}

\author{
Paul Dennison* \\ Independent Researcher, London, United Kingdom \\ *Correspondence: \\ pd@pauldennisonpsychotherapy.co.uk
}

\begin{abstract}
This commentary explores the Covid-19 pandemic against the background of global climate change. We live in interdependence with our environment at a hierarchy of scales; from the fertilised egg in the womb, the newborn with mother, children with parents and family, adults in societies, and finally societies in interdependence with our planet. We consider the processes of interaction at hierarchical levels as forms of "communication", often deeply unconscious and nonverbal, to better understand what we might be dealing with during this present crisis, and how to respond in addition to the obvious medical and epidemiological considerations.
\end{abstract}

\section{Introduction}

It may be no coincidence that with the Covid-19 pandemic we are witnessing human suffering on a global scale, at the same time that we face catastrophic effects of climate change caused by our negligence as human beings towards our environment and planet. Recent developments in theoretical neuroscience (Friston, 2010; Seth and Friston, 2016) highlight the interactive nature by which biological entities, in particular human beings, are influenced by their environment and in turn through their actions influence and potentially change that same environment in a normally never-ending dance. At the level of individuals in society, the arena for this dance is the outer sensory world from which we receive input via our senses, as well as from our body, all processed in relation to memory via incredibly fast unconscious loops, leading to actions and behaviour; supporting and largely creating the personal experience of "I". In this paper we refer to this level of hierarchical "communication" to constitute the human default everyday consciousness.

A quite different research project (Dennison, 2019) examining changes in brain activity during a little known but ancient form of Buddhist meditation known as jhāna, has shown that if a meditator disengages his/her personal "I" from their default sensory consciousness, then a different pattern of brain activity along a vertical brain-body axis develops, with jhāna consciousness experienced subjectively as a more timeless embodied "presence".

Taken together, these results accord with theoretical predictions that life as a whole is supported by nested hierarchical systems, each delineated by a "Markov blanket" (Kirchoff et al., 2018). The most primary system for our everyday experience of "I am", and "I do", is our relation to the external sensory world, which we term the human default (sensory) consciousness. Inner to this is then the brain-body system revealed in the special case of jhāna meditation, with an accompanying consciousness experienced as presence rather that the default sensory consciousness of "this" or "that". Which then leads us to consider the outer encompassing level of planet Earth-societies, and whether at this global scale we are witnessing breakdown of the outermost Markov blanket evidenced by parallel chaotic and destructive climate change at the outer level, and the recent destructive Covid-19 pandemic affecting the inner societal level. 


\section{Supplementary Material: Graphical Abstract}

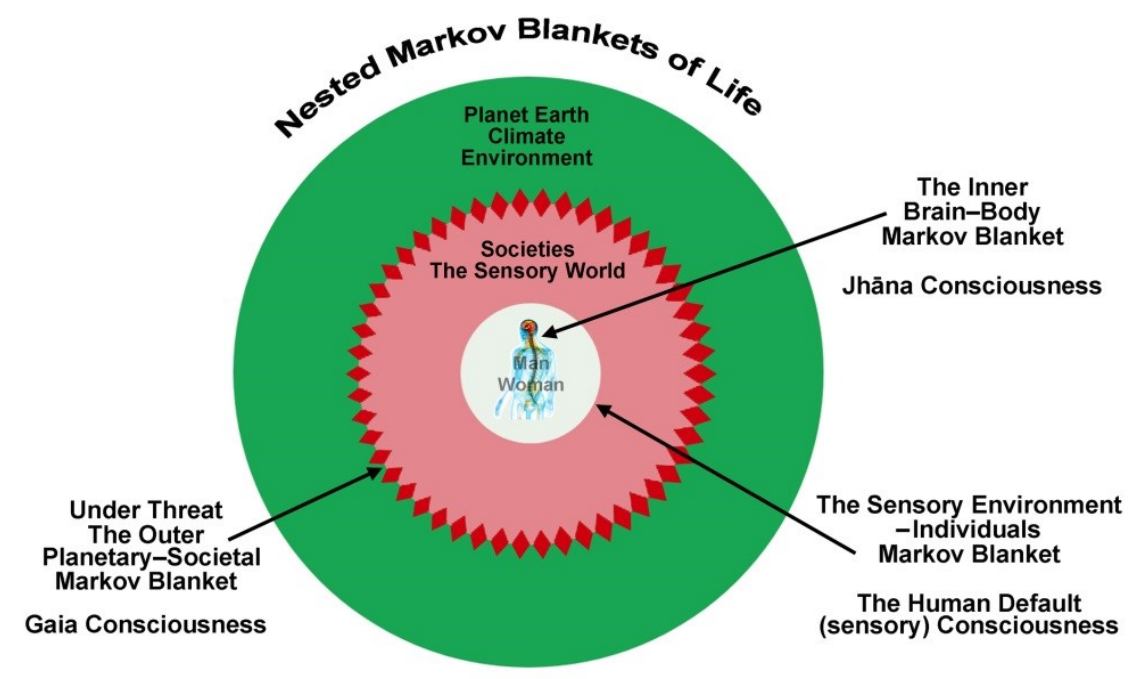

\section{Free Energy and Active Inference}

Beginning in 2010, Karl Friston's free energy theory rapidly evolved into detailed models of "active inference" with far-reaching implications for understanding consciousness and how as biological self-organising systems we adapt to living in a sensory world (Friston, 2010; Seth and Friston, 2016). In brief, our brain receives sensory input from our environment, in particular via sight, hearing and touch, and from our body via biological and nervous system signals, all then processed in relation to past experiences held in memory, leading to actions. However, this is far from a passive process; there has to be an "agent" to govern the motivation or choice of action, and theory predicts that the choice, determined by incredibly fast predictive processing at an unconscious level, will be that which makes the most efficient use of available energy, at the same time as minimising "surprise" or unexpected shocks to the system. The "system" being of course, our-self.

\section{Sensory Consciousness and Jhāna Meditation}

In parallel with these theoretical developments in neuroscience, an unrelated research project (Dennison, 2019) was underway to explore brain activity during a little-known and some would say esoteric form of Buddhist meditation known as jhāna (Cousins, 1973; Gunaratana, 1980). Unlike more familiar forms of meditation such as mindfulness, jhāna meditation seeks to penetrate to the deepest levels of consciousness and perception in order to understand the roots of our sense of self and, in Buddhist terms, the nature and propensity towards human suffering. To develop jhāna, a state of inner-directed intense absorption, meditators are required to disengage from their everyday sensory consciousness towards states of increasing simplicity, letting go of habitual cognitive processes of naming and discrimination that support the default consciousness of "this" or "that". The resulting subjective experience is most often described by meditators as embodied presence.

The startling results of this study of experienced lay Western meditators demonstrated profound disruption of brain networks to an extent never previously seen in neuroscience. The primary networks disrupted were those familiar in neuroscience that govern attention, and that are central to the active predictive inference processes described above. It was on this basis that Dennison (2019) explicitly named a "human default (sensory) consciousness" to describe those brain networks disrupted during jhāna meditation, suggesting further that this default consciousness is precisely that organisation, the "agent" or subject, described by Friston and others in the theoretical free 
energy and active-inference literature.

The most remarkable finding of the meditation study, however, was the emergence of a new axis of consciousness during the deeper levels of jhāna. As jhāna developed for the most experienced meditators, the complex and widespread brain networks supporting their default sensory consciousness gradually faded into quiescence to be replaced by a vertical axis of consciousness focused at an intense "hotspot" at the crown of the head known to have high connectivity down through core regions of the brain to the brain-stem, and hence into the vagus sympathetic and parasympathetic nerve systems of the body.

This "jhāna consciousness", as opposed to the default sensory consciousness, was characterised by a much slower time scale-factor of $\sim 10-30$ secs, compared to the $\sim 100 \mathrm{~ms}$ of our everyday consciousness (corresponding to the minimum reaction time), presumably related to a more timeless subjective experience during jhāna. Subsequent research on heart-rate variability, before and during meditation, is currently underway to confirm a deep metabolic integration evidenced by enhanced parasympathetic nervous system activity in the body, in turn related to the sense of embodied peace in this form of meditation.

\section{Existence, Identity and Markov Blankets}

A highly creative extension of the free-energy and predictive active-inference literature introduced the concept of the Markov blanket. For anything to "exist", whether at the smallest atomic or cellular level, up through hierarchies of biological organisms, human beings and even societies, it has to be distinguished from its environment by a boundary, which in mathematical and statistical probabilistic theory is termed a Markov blanket (an evocative term coined by Judea Pearl, a computer scientist and philosopher in 1988). Without

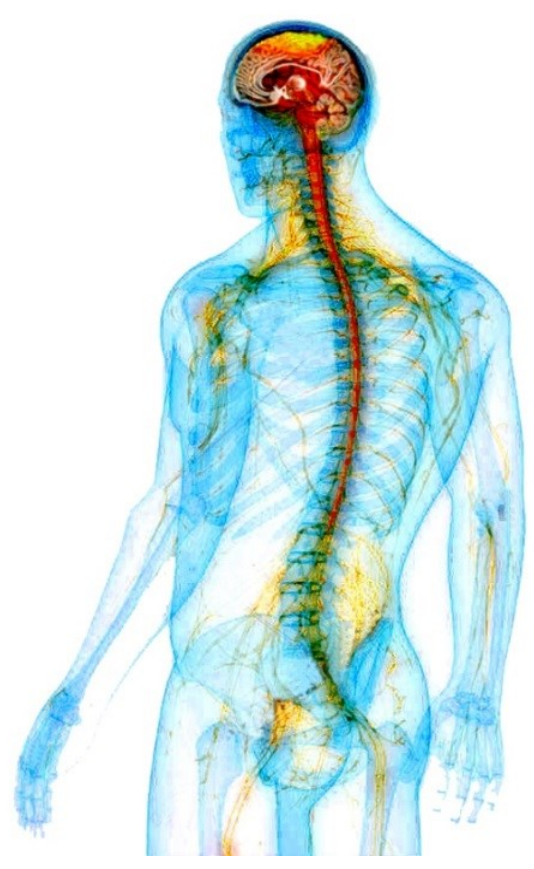

Figure 1 A conceptual illustration of the jhāna axis from the crown of the head down into the nervous systems of the body a boundary of enclosure, a prototype entity would simply dissipate in accordance with the laws of entropy and thermodynamics (see Kirchoff et al., 2018, "The Markov Blankets of Life").

Furthermore, theory predicts the existence of "nested" Markov blankets at different hierarchical levels of organisation. Thus the Markov blanket for our human default consciousness defines the boundary between the outer environment and the inner person, linking sensory information to action via the processes of adaptive and predictive active inference; supporting our sense of "I". For the special case of jhāna meditation, in contrast, research suggests that as a meditator disengages from the outer sensory world, and as the "I" of default sensory consciousness is replaced by a new and simpler jhāna consciousness, more appropriately called "presence", a new, inner Markov blanket becomes active defining the interactions between brain-body systems. It is entirely possible that the core of the sense of presence that characterises jhâna consciousness lies in the intermediate core brain-stem regions rather than the cortical structures that underpin sensory consciousness. This hypothesis is similar to that of Solms (2019) who describes affect as the "elemental form of consciousness", with its physiological mechanisms located in the upper brain-stem.

\section{Covid-19 and Climate Change: Breakdown of the Global Markov Blanket}

Much research is already underway into the effects of climate change on pathogens, transmission 
and epidemiology, and our intention here is to add a systems-theoretic perspective based on the concept of Markov blankets. Beyond the Markov blanket that defines the individual and his/her sensory consciousness, or the inner Markov blanket that we suggest underpins jhāna consciousness, an outer Markov blanket must exist to define the boundary and interactions between global systems on the outside, and societies within. Such a boundary is implicit to Friston's (2010) free-energy theory and subsequent elaborations, as well as the extensive statistical probabilistic theoretical work supporting the concept of the Markov blanket.

Now since it is an integral feature of the theory of Markov blankets that over time "an organism [i.e. the inner level] will become a model of its environment" (Kirchoff et al., 2018), then we might expect similar chaotic and destructive processes to appear in societies as the counterpart to accelerating levels of chaotic climate breakdown.

What might "similar" mean in practice? The interactions between outer and inner at each level of hierarchy can be regarded as forms of communication, so that at the level of sensory consciousness, for example, communication is predominantly via our familiar verbal language, where distortions or breakdowns across the society-individual Markov blanket result in verbally expressed conflicts, both outer and inner, as described in psychoanalytic models of language and defence (Freud, 1895; Dennison, 1997). While the processes of adaptive active inference that underpin this everyday default sensory consciousness by and large lead to a remarkably stable dynamic low-energy balance in the brain's neural activity, it is an open question as to the stability of the outer global-societal Markov blanket. Given its necessarily much longer time scale-factor (months or years), what kind of communication can we imagine between the outer and inner? Patterns and themes, or the symbolic level, might be the most obvious answer in attempting to formulate what the above quote, that over time "[the inner] will become a model of its environment", might mean. Indeed, Oseledchik et al. (2016) suggest that fractal processes can govern the transmission and reconfiguration of meaning across Markov blankets, which would support this hypothesis.

It may not then be coincidence that alongside the catastrophic out-of-control fires and flooding of 2019-20, undoubtedly related to climate change and global warming, that we find ourselves suddenly afflicted by a quite new form of invasive and deadly virus; one that causes high fever, and where the barriers of the human immune system become as ineffective as a city's flood defences in being unable to prevent congestive lung disease and death in those most vulnerable. The language of correspondences between "out-of-control fires" and "fever", or between floods and pneumonia, reminds us of distorted (tangential) speech in schizophrenia, a result of disengagement from reality similar to Freud's (1924) description of how words become "things" in the approach towards psychosis. Indeed, the present parallels between climate breakdown and the Covid-19 pandemic may indicate something akin to a "psychotic breakdown" of the global-societal Markov blanket.

Theoretical work on adaptive active inference requires that in order to prevent catastrophic breakdown at some point in the future, ultimately "to remain alive", the inner system must not only "be able to make inferences about the outcomes of its own actions", but must equally "possess a generative model with temporal depth" to allow a range of options to be sampled to "avoid crossing terminal species-specific phase boundaries" (Kirchoff et al., 2018; my emphasis). Since the range of options are explored via very fast unconscious looped processes, what are the factors that influence choice of option, and is it possible to avoid a future and even impending catastrophic terminal phase boundary?

\section{Cross-discipline Options and Lessons from Psychoanalysis and Buddhist Practice}


Taking the example of the human default sensory consciousness, both psychoanalytic and Buddhist models would agree that liking versus disliking, or greed versus aversion, even hatred, are primary motivators for behaviour and choice of actions (Dennison, 2019). Starting with Freud (1895), the approach of psychoanalysis has been to uncover processes of denial and defence rooted in fear or craving for satisfaction, effectively to make the unconscious conscious; processes described by Holmes and Nolte (2019) in the context of the more recent free-energy and active inference literature. Similarly, Buddhism sees the cause of human suffering as due to craving, in particular greed or ill-will inherent in attachment to a sensory world (Harvey, 2012), hence the central importance in Buddhist practice of jhāna meditation to disengage, at least temporarily, from enmeshment in the sensory world to achieve a less-conflicted perspective.

On the larger global scale of human species survival, we can no longer avoid the evidence of breakdown at both the outer (planetary) and inner (societal) levels. In short, we no longer have a safe Markov blanket. On smaller scales there have already been examples of species extinction related to climate change that have already happened; for example Pound et al.'s (2006) study of widespread amphibian extinctions driven by global warming. More recently $\mathrm{Wu}$ et al. (2016) reviewed evidence for climate change effects on pathogens, hosts and transmission processes, noting that on the extreme global scale the 1997-98 and 2015-16 El Ninos led to outbreaks of malaria and cardio-pulmonary syndromes; episodes of severe flooding were related to outbreaks of cryptosporidium, typhoid, cholera, lymphatic disease and haemorrhagic fever; heatwaves to West Nile fever and increased morbidity and mortality from infectious respiratory diseases; and drought to hantavirus pulmonary syndrome (HPS), increased risk of West Nile virus and transmission of the St. Louis encephalitis virus. Also, quite apart from the occurrence of new pathogens, and in the case of flu-like viruses their apparent increased ability to mutate and hence frustrate immune-system response, the immune system response itself has recently been demonstrated to be less effective as a result of increased seasonal and rapid climate variability (Liu et al., 2020). Yet the evidence from such lesser catastrophes in the past is that we do not take responsibility for destructive behaviours that influence climate change, or change those behaviours.

An intriguing side-effect of the Covid-19 pandemic is the visible evidence of much reduced global pollution as industries are forced into inactivity, with less fossil fuel usage, much reduced air travel, and so on. Indeed, some might wonder whether we are being shown by Gaia what could and ought to be done; in which case it might be well to bear in mind that in Greek mythology Gaia was preceded by Chaos, and whether the terminal phase-transition mentioned earlier might be a return to chaos. The real test will of course come when the crisis passes; will the drivers of greed re-emerge, even heightened in a rush to make up losses? Or can societies pause to reflect, to resist the habitual unconscious processes that led us to this point; particularly the driver of greed, the greed for more and more profit, the greed to outstrip one's neighbour via global competition, no matter the cost.

It is not the suggestion of this paper that jhāna meditation might be any kind of remedy or balancing factor, particularly since its experience usually requires years of practice. However, the starting point to eventually develop jhāna is mindfulness, which is not difficult to practice, as evidenced by its value in easing mild to moderate anxiety and depression in mental health (Huang, S.-L et al., 2015; Dunning et al., 2018). In Buddhist practice, mindfulness means attending to the present, but to develop its full potential requires a degree of discrimination in resisting attachment to past patterns, and learning to deal with greed and fear that usually underlie wrong choices of action. This accords with anecdotal evidence from those like this author familiar with its application in mental health, that the therapeutic relationship (which basically equates to healthy, mature communication) is a key factor in fostering reflective discrimination. In the aftermath of the current crisis, we might hope for a reflective and mindful space where societies as a whole can learn from these extremely painful experiences to moderate behaviour in the future. The challenge will be to encourage and demonstrate the potential benefits of reflective collaborative behaviour, before old habits re- 
establish themselves. The existential shock of the Covid-19 pandemic may provide a small window of opportunity.

Furthermore, from the above discussion of active inference, it is difficult to imagine how the inner pole of the global-societal Markov blanket can relate consistently and stably to the outer planetary level without such a shared endeavour. If societies, as the inner pole of an adaptive active-inference system, are to be able to "make inferences about the outcomes of [their] own actions", they are therefore implicitly required to function as a united agency. Here, too, both Buddhism and psychoanalysis would recognise common ground; that ultimately we are no different to our neighbour, we are born and inevitably will die, and we all live the raw and rich ups and downs, satisfactions and frustrations, of life in between. This common ground is the capacity for compassion.

A final caution. If indeed we are witnessing a breakdown in the global-societal Markov blanket with similarities to a denial of reality in psychosis, then we might bear in mind lessons from psychiatry. The distortions of communication, language and behaviour shown by psychiatric patients in the grip of a psychotic episode are often so out of touch with reality that they elicit fear and avoidance in onlookers. If fear is resisted, however, including the pervasive fear triggered by Covid-19, meaning may be found in the language and communications of psychosis (Laing and Esterson, 1964), and Gaia may indeed be conveying a message through the symbolic and fractal parallels between climate change and Covid-19 that we need to take seriously.

\section{Author Contributions}

The author confirms being the sole contributor of this work and has approved it for publication.

\section{Conflict of Interest Statement}

The author declares that this research was conducted in the absence of any commercial or financial relationships that could be construed as a potential conflict of interest.

\section{References}

Cousins, L.S. (1973) Buddhist jhāna: its nature and attainment according to Pali sources. Religion 3, 115-31.

Dennison, P. A. (2019). The human default consciousness and its disruption: insights from an EEG study of Buddhist jhāna meditation. Front. Hum. Neurosci. 13:178. doi: 10.3389/fnhum.2019.00178

Dennison, P. (1997) Language and defence and the self-representation. Essay, Regent's University, School of Psychotherapy, doi: 10.13140/RG.2.1.4233.4885

Dunning, D. et al. (2018) The effects of mindfulness-based interventions on cognition and mental health in children and adolescents: a meta-analysis of randomised controlled trials. J. Child Psychol. and Psychiatry, 60(3):244-58. doi: 10.1111/jcpp. 12980

Freud, S. (1895) Project for a Scientific Psychology. Standard Edition, Vol. 1. London: Hogarth Press.

Freud, S. (1924) Neurosis and Psychosis. The Standard Edition of the Complete Psychological Works of Sigmund Freud, Volume XIX. London: Karnac.

Friston, K. (2010) The free-energy principle: a unified brain theory? Nature Revs Neurosci. 11:127-39. doi: $10.1038 / \mathrm{nrn} 2787$

Gunaratana, H. (1980) A Critical Analysis of the jhānas in Theravada Buddhist meditation. PhD dissertation, Washington: The American University. Published (1985) as The Path of Serenity and Insight: An Explanation of Buddhist Jhānas. Delhi: Motilal Banarsidass.

Harvey, P. (2012) An Introduction to Buddhism. Cambridge: Cambridge University Press.

Holmes, J. and Nolte, T. (2019) "Surprise" and the Bayesian brain: implications for psychotherapy theory and practice. Front. Psychol. 10. doi: 10.3389/fpsyg.2019.00592 
Huang, S.-L., Li, R.-H.,Huang, F.-Y. and Tang, F.-C. (2015) Th potential for mindfulness-bases intervention in workplace mental health promotion: results of a randomised controlled trial. PLOS ONE 10(9). doi: 10.1371/journal.pone.0138089

Kirchhoff, M., Parr, T., Palacios, E., Friston, K., Kiverstein, J. (2018). The markov blankets of life: autonomy, active inference and the free energy principle. J. R. Soc. Interface. doi: 10.1098/rsif.2017.0792

Laing, R.D. and Esterson, A. (1964) Sanity, Madness and the Family. London: Penguin Books.

Liu, Q. et al. (2020) Changing rapid weather variability increases influenza epidemic risk in a warming climate. Environ. Res. Letters, 15: 044004. doi: 10.1088/1748-9326/ab70bc

Oseledchik, M., Ivleva, M. and Ivleva, V. (2017) The fractal nature of implicit knowledge. Advances in Social Science, Education and Humanities Research, Vol. 144. $3^{\text {rd }}$ International Conference on Arts, Design and Contemporary Education. Paris: Atlantis Press. doi: 10.2991/cesses-19.2019.6

Pearl, J. (1988) Probabilistic Reasoning in Intelligent Systems: Networks of Plausible Inference. San Mateo CA: Morgan Kaufmann.

Pounds, J.A. et al. (2006) Widespread amphibian extinctions from epidemic disease driven by global warming. Nature 439. doi: 10.1038 /nature 04246

Seth, A.K. and Friston, K.J. (2016) Active interoceptive inference and the emotional brain. Phil. Trans. R. Soc. B 371: 20160007. doi: 10.1098/rstb.2016.0007

Solms, M. (2019) The hard problem of consciousness and the free energy principle. Front. Psych. 9:2714. doi: $10.3389 /$ fpsyg.2018.02714 